\title{
Microbiological profile of ozenoxacin
}

\author{
Ian Morrissey ${ }^{1}$, Rafael Cantón 2,3 , Jordi Vila3,4,5, Domingo Gargallo-Viola ${ }^{6}$, Ilonka Zsolt ${ }^{*, 7}$, \\ Maria Garcia-Castillo ${ }^{2,3}$ \& Yuly López ${ }^{5}$ \\ ${ }^{1}$ IHMA Europe Sàrl, Monthey, Switzerland \\ ${ }^{2}$ Servicio de Microbiología, Hospital Universitario Ramón y Cajal \& Instituto Ramón y Cajal de Investigación Sanitaria (IRYCIS), \\ Madrid, Spain \\ ${ }^{3}$ Red Española de Investigación en Patología Infecciosa (REIPI), Instituto de Salud Carlos III, Madrid, Spain \\ ${ }^{4}$ Microbiology Service, Biomedical Diagnostic Center, Hospital Clinic, University of Barcelona, Spain \\ ${ }^{5}$ Institute of Global Health of Barcelona (ISGlobal), Barcelona, Spain \\ ${ }^{6}$ ABAC Therapeutics, Barcelona, Spain \\ ${ }^{7}$ Medical Department, Ferrer Internacional, Barcelona, Spain \\ *Author for correspondence: izsolt@ferrer.com
}

Aim: To explore the antibacterial spectrum of ozenoxacin and compare its in vitro activity with that of other antibacterial agents. Materials \& methods: In 2010, 10,054 isolates were collected from 128 centers worldwide. Minimum inhibitory concentrations against Gram-positive and Gram-negative isolates were determined for 23 and 13 antibacterial agents, respectively. Results: Ozenoxacin exhibited high in vitro activity against susceptible, and methicillin- or levofloxacin-resistant, Gram-positive bacteria. Ozenoxacin was one or two dilutions less active against Enterobacteriaceae isolates, except for Escherichia coli, than other quinolones. Conclusion: Ozenoxacin is a potent antimicrobial agent mainly against susceptible and resistant strains of Gram-positive isolates (staphylococci and streptococci), and shows activity against some Gram-negative isolates.

First draft submitted: 26 March 2019; Accepted for publication: 9 May 2019; Published online: 28 May 2019

Keywords: antibacterial activity $\bullet$ clinical isolates $\bullet$ Gram negative $\bullet$ Gram positive $\bullet$ ozenoxacin $\bullet$ respiratory tract infections • skin and soft tissue infections

Impetigo is a common bacterial skin infection affecting children and adults that is caused by Staphylococcus aureus or Streptococcus pyogenes. It is the most common bacterial skin infection in children aged 2-5 years [1,2].

Impetigo is commonly treated with topically applied antibacterial agents such as mupirocin, fusidic acid and retapamulin [1-5]. A recent evidence-based review supported the clinical efficacy of mupirocin and fusidic acid for treatment of impetigo [5]. However, in some cases, development of resistant isolates limits the uses of these agents [1,5].

An alternative option for treating impetigo in children and adults is a $1 \%$ topical cream formulation of ozenoxacin, a nonfluorinated quinolone with bactericidal activity [6], which was recently approved in several countries worldwide [7-10]. Approval of ozenoxacin was granted based on demonstrable clinical benefit in two large Phase III trials of impetigo involving adults and children from 2 months of age [11,12]. Rapid eradication of bacterial load is important in impetigo to hasten symptom resolution and limit person-to-person transmission of infection.

Early in vitro characterization of ozenoxacin found that it was 3- to 321-fold more active than other quinolones against quinolone-susceptible and quinolone-resistant Gram-positive bacteria [13]. A subsequent and more comprehensive study showed that ozenoxacin has potent antibacterial activity against staphylococci and streptococci, irrespective of their levofloxacin susceptibility status [14].

This article presents additional surveillance study data generated during development of ozenoxacin. The antibacterial activity of ozenoxacin against Gram-positive and Gram-negative clinical isolates was compared with that of other topical and systemic antibiotics used in the treatment of skin and soft tissue infections (SSTIs). As it had yet to be decided at the time of the study whether ozenoxacin was to be developed as a systemic (oral or intravenous) and/or topical formulation, in order to ascertain its full microbiological profile, its antibacterial 
activity was also investigated against Gram-positive and Gram-negative bacteria causing respiratory tract infections (RTIs) and urinary tract infections (UTIs).

\section{Methods}

\section{Clinical isolates collection}

During 2010, a total of 10,054 bacterial isolates were collected from 128 centers, with some sites collecting isolates from more than one hospital. Centers were located in the USA $(n=28)$; Germany $(n=15)$; France $(n=8)$; Turkey $(\mathrm{n}=8)$; the UK $(\mathrm{n}=7)$; Spain $(\mathrm{n}=6)$; Italy $(\mathrm{n}=5)$; Belgium, Canada, Greece, Portugal and South Africa $(\mathrm{n}=4$ each); Argentina, Australia, Austria, Czech Republic, Japan, The Philippines, Slovak Republic, Thailand ( $\mathrm{n}=2$ each); and Hong Kong, Hungary, Ireland, Israel, Malaysia, New Zealand, Poland, Romania, South Korea, Sweden, Switzerland, Taiwan and The Netherlands ( $\mathrm{n}=1$ each).

Isolates were derived from SSTIs (44.8\%), RTIs (38.1\%), UTIs $(1.3 \%)$, unknown $(8.4 \%)$ or other $(7.5 \%)$ sources. Taxonomic classification of isolates is summarized in Table 1. Isolates were identified following standard microbiological methods, including biochemical test and mass spectrometry (MALDI TOF MS).

\section{Minimum inhibitory concentration determination}

Minimum inhibitory concentration (MIC) was determined using broth microdilution methods as recommended by the Clinical and Laboratory Standards Institute (CLSI) [15-17].

Susceptibility was determined using breakpoints set by the CLSI [15,17], except that British Society for Antimicrobial Chemotherapy (BSAC) breakpoints were used for fusidic acid and mupirocin against staphylococci [18].

S. aureus ATCC 29213, S. pneumoniae ATCC 49619, Escherichia coli ATCC 25922 and E. coli ATCC 35218 isolates were used for quality control. All quality control results were within the ranges specified by the CLSI [17,19].

Concentrations of antibacterial agent tested for each isolate are shown in Table 2.

\section{Results}

\section{Classification of clinical isolates}

Gram-positive bacteria included in the study as principle pathogens were: coagulase-negative staphylococci (CNS), S. aureus, $\beta$-haemolytic streptococci, viridans streptococci, $S$. pneumoniae and enterococci (Table 1). A high proportion of Gram-positive isolates (69.5\% of CNS [618/889]; 63.3\% of S. aureus [1518/2398]; 78.8\% of $\beta$-haemolytic streptococci [953/1210]; $66.2 \%$ of viridans streptococci [353/533]; and 50.6\% of enterococci [84/166]) were derived from SSTIs.

Gram-negative bacteria included in the study as principle pathogens were: Enterobacteriaceae $(\mathrm{n}=3190)$, Haemophilus spp. $(\mathrm{n}=548)$ and Moraxella catarrhalis $(\mathrm{n}=180)$ (Table 1). A high proportion of Gram-negative isolates classified as Haemophilus spp. $(95.8 \%$; 525/548) or M. catarrhalis $(93.9 \%$; 169/180) were derived from RTIs. Similarly, a high proportion of Gram-positive S. pneumoniae isolates $(90.9 \%$; 843/927) were derived from RTIs.

\section{Comparative antimicrobial activity Gram-positive bacteria}

Staphylococci

Ozenoxacin was highly active against staphylococci with a $\mathrm{MIC}_{90}$ of $0.25 \mathrm{mg} / \mathrm{l}$ against $S$. aureus and all CNS isolates (Table 3). In general, ozenoxacin had high activity against Gram-positive bacteria irrespective of their resistance/susceptibility to methicillin or nonsusceptibility/susceptibility to levofloxacin.

A comparison of $\mathrm{MIC}_{90}$ values in SSTI-derived isolates (Table 4) demonstrated that ozenoxacin $(0.5 \mathrm{mg} / \mathrm{l})$ was more active against CNS isolates than 19 of 22 comparator agents tested including vancomycin and linezolid $(2 \mathrm{mg} / \mathrm{l})$; moxifloxacin $(4 \mathrm{mg} / \mathrm{l})$; amoxicillin-clavulanate and teicoplanin $(8 \mathrm{mg} / \mathrm{l})$; levofloxacin $(>8 \mathrm{mg} / \mathrm{l})$; fusidic acid and trimethoprim-sulfamethoxazole $(16 \mathrm{mg} / \mathrm{l})$; meropenem (>16 mg/l); ampicillin, neomycin and tetracycline $(32 \mathrm{mg} / \mathrm{l})$; ceftriaxone and clarithromycin (>32 mg/l); penicillin and piperacillin/tazobactam $(64 \mathrm{mg} / \mathrm{l})$; and mupirocin, ceftazidime and cefuroxime $(>64 \mathrm{mg} / \mathrm{l})$. Retapamulin was the only comparator tested to have a $\mathrm{MIC}_{90}$ value $(0.12 \mathrm{mg} / \mathrm{l})$ lower than that of ozenoxacin, while tigecycline and daptomycin each had a $\mathrm{MIC}_{90}$ value $(0.5 \mathrm{mg} / \mathrm{l})$ equivalent to that of ozenoxacin.

Against $S$. aureus specifically (Table 4), ozenoxacin was equally as active as mupirocin, retapamulin, tigecycline and trimethoprim-sulfamethoxazole $\left(\mathrm{MIC}_{90}\right.$ of $\left.0.25 \mathrm{mg} / \mathrm{l}\right)$, and was more active than 18 of 22 comparator 
Table 1. Categorization of isolates $(n=10,054)$ from skin and soft tissue infections, respiratory tract infections, urinary tract infections and other or unknown sources ( $\geq 10$ in $\geq 1$ category).

\begin{tabular}{|c|c|c|c|c|c|c|c|}
\hline Group & Pathogen & SSTI & RTI & UTI & Unknown & Other & Total \\
\hline \multicolumn{8}{|l|}{ Gram positive } \\
\hline \multirow[t]{8}{*}{ Coagulase-negative staphylococci (CNS) } & All CNS & 618 & 106 & 1 & 146 & 18 & 889 \\
\hline & Staphylococcus capitis & 30 & 8 & & 12 & 3 & 53 \\
\hline & Staphylococcus epidermidis & 365 & 47 & & 86 & 9 & 507 \\
\hline & Staphylococcus haemolyticus & 76 & 18 & & 11 & 1 & 106 \\
\hline & Staphylococcus hominis & 48 & 20 & & 22 & 4 & 94 \\
\hline & Staphylococcus lugdunensis & 53 & 4 & & 6 & & 63 \\
\hline & Staphylococcus pettenkoferi & 10 & 1 & & 3 & & 14 \\
\hline & Staphylococcus warneri & 7 & 4 & & 4 & 1 & 16 \\
\hline Staphylococcus aureus & Staphylococcus aureus & 1518 & 666 & 1 & 196 & 17 & 2398 \\
\hline \multirow[t]{4}{*}{$\beta$-haemolytic streptococci } & All $\beta$-haemolytic streptococci & 953 & 118 & 5 & 127 & 7 & 1210 \\
\hline & Streptococcus agalactiae & 271 & 11 & 5 & 37 & 3 & 327 \\
\hline & Streptococcus dysgalactiae & 172 & 28 & & 21 & 1 & 222 \\
\hline & Streptococcus pyogenes & 507 & 78 & & 69 & 3 & 657 \\
\hline \multirow[t]{10}{*}{ Viridans streptococci } & All Viridans streptococci & 353 & 64 & 1 & 108 & 7 & 533 \\
\hline & Streptococcus anginosus & 91 & 11 & & 14 & 1 & 117 \\
\hline & Streptococcus constellatus & 60 & 6 & & 4 & & 70 \\
\hline & Streptococcus gallolyticus & 38 & 2 & 1 & 19 & & 60 \\
\hline & Streptococcus intermedius & 15 & 4 & & 3 & & 22 \\
\hline & Streptococcus mitis & 29 & 13 & & 21 & 2 & 65 \\
\hline & Streptococcus oralis & 39 & 8 & & 12 & 1 & 60 \\
\hline & Streptococcus parasanguinis & 18 & 4 & & 4 & & 26 \\
\hline & Streptococcus salivarius & 23 & 7 & & 10 & 1 & 41 \\
\hline & Streptococcus sanguinis & 10 & 1 & & 7 & 1 & 19 \\
\hline - Streptococcus pneumoniae & Streptococcus pneumoniae & 63 & 843 & & 18 & 3 & 927 \\
\hline \multirow[t]{2}{*}{ Enterococci } & All Enterococci & 84 & 8 & 41 & 26 & 7 & 166 \\
\hline & Enterococcus faecalis & 81 & 7 & 39 & 26 & 6 & 159 \\
\hline \multicolumn{8}{|l|}{ Gram negative } \\
\hline \multirow[t]{13}{*}{ Enterobacteriaceae } & All Enterobacteriaceae & 895 & 1329 & 70 & 208 & 688 & 3190 \\
\hline & Citrobacter freundii & 50 & 33 & 8 & 4 & 38 & 133 \\
\hline & Citrobacter koseri & 40 & 34 & 10 & 1 & 34 & 119 \\
\hline & Klebsiella aerogenes & 54 & 45 & 4 & 4 & 51 & 158 \\
\hline & Enterobacter cloacae & 73 & 74 & 2 & 5 & 65 & 219 \\
\hline & Escherichia coli & 287 & 508 & 17 & 5 & 234 & 1051 \\
\hline & Klebsiella oxytoca & 51 & 100 & 6 & & 40 & 197 \\
\hline & Klebsiella pneumoniae & 94 & 350 & 11 & 2 & 87 & 544 \\
\hline & Morganella morganii & 144 & 44 & 3 & 2 & 40 & 233 \\
\hline & Salmonella spp. & 15 & 5 & 3 & 106 & 34 & 163 \\
\hline & Serratia marcescens & 61 & 117 & 4 & 6 & 44 & 232 \\
\hline & Shigella flexneri & & 1 & 2 & 32 & 5 & 40 \\
\hline & Shigella sonnei & 3 & 2 & & 34 & 2 & 41 \\
\hline \multirow[t]{5}{*}{ Other Gram-negative bacteria } & Haemophilus spp. & 11 & 525 & & 3 & 9 & 548 \\
\hline & Haemophilus influenzae & 3 & 458 & & 2 & 7 & 470 \\
\hline & Haemophilus parainfluenzae & 8 & 62 & & 1 & 2 & 73 \\
\hline & Moraxella catarrhalis & 4 & 169 & 6 & & 1 & 180 \\
\hline & Neisseria gonorrhoeae & 2 & & 7 & 4 & & 13 \\
\hline
\end{tabular}


Table 2. Concentrations of antibacterial agent tested for each bacteria.

\begin{tabular}{|c|c|c|c|c|c|c|}
\hline \multirow[t]{2}{*}{ Antibacterial } & \multicolumn{6}{|c|}{ Antibacterial range tested $(\mathrm{mg} / \mathrm{l})$} \\
\hline & $\begin{array}{l}\text { Staphylococci and } \\
\text { Enterococci }\end{array}$ & $\begin{array}{l}\text { Streptococcus spp. } \\
\text { (other than } S . \\
\text { pneumoniae) }\end{array}$ & $\begin{array}{l}\text { Streptococcus } \\
\text { pneumoniae and } \\
\text { Moraxella catarrhalis }\end{array}$ & Enterobacteriaceae & Haemophilus spp. & Neisseria gonorrhoeae \\
\hline Mupirocin & $0.03-64$ & $0.03-64$ & & & & \\
\hline Fusidic acid & $0.03-64$ & $0.03-64$ & & & & \\
\hline Levofloxacin & $0.004-8$ & $0.03-64$ & $0.03-64$ & $0.004-8$ & $0.008-16$ & $0.004-4$ \\
\hline Ciprofloxacin & & & & $0.002-4$ & & $0.004-4$ \\
\hline Ampicillin & $0.015-32$ & $0.008-16$ & $0.015-32$ & $0.015-32$ & $0.008-16$ & \\
\hline $\begin{array}{l}\text { Amoxicillin- } \\
\text { clavulanate }\end{array}$ & $0.015-32$ & $0.008-16$ & $0.008-16$ & $0.015-32$ & $0.015-32$ & \\
\hline Cefuroxime & $0.03-64$ & $0.008-16$ & $0.008-16$ & $0.03-64$ & $0.015-16$ & $0.008-1$ \\
\hline Linezolid & $0.015-32$ & $0.015-32$ & $0.015-32$ & & & \\
\hline Meropenem & $0.008-16$ & $0.008-16$ & $0.008-16$ & $0.004-8$ & $0.008-16$ & \\
\hline Neomycin & $0.03-64$ & $0.03-64$ & & $0.03-64$ & & \\
\hline Penicillin G & $0.03-64$ & $0.008-16$ & $0.008-16$ & & $0.008-16$ & $0.015-8$ \\
\hline Piperacillin & & & & $0.06-128$ & $0.06-128$ & \\
\hline $\begin{array}{l}\text { Piperacillin- } \\
\text { tazobactam }\end{array}$ & $0.03-64$ & $0.015-32$ & $0.03-64$ & $0.06-128$ & $0.03-64$ & \\
\hline Retapamulin & $0.008-16$ & $0.008-16$ & & & & \\
\hline Teicoplanin & $0.015-32$ & $0.015-32$ & $0.015-32$ & & & \\
\hline Tetracycline & $0.03-64$ & $0.03-64$ & $0.03-64$ & & $0.03-64$ & $0.015-8$ \\
\hline
\end{tabular}

antibacterial agents tested that had $\mathrm{MIC}_{90}$ values of $0.5 \mathrm{mg} / \mathrm{l}$ (fusidic acid and daptomycin); $1 \mathrm{mg} / \mathrm{l}$ (teicoplanin and vancomycin); $2 \mathrm{mg} / \mathrm{l}$ (linezolid); $4 \mathrm{mg} / \mathrm{l}$ (moxifloxacin); $>8 \mathrm{mg} / \mathrm{l}$ (levofloxacin); $16 \mathrm{mg} / \mathrm{l}$ (amoxicillin/clavulanic acid and meropenem); $32 \mathrm{mg} / \mathrm{l}$ (tetracycline); $>32 \mathrm{mg} / \mathrm{l}$ (ampicillin, ceftriaxone and clarithromycin); $64 \mathrm{mg} / \mathrm{l}$ (penicillin and piperacillin-tazobactam); and $>64 \mathrm{mg} / \mathrm{l}$ (ceftazidime, cefuroxime and neomycin).

Ozenoxacin was consistently among the most active antibacterial agents (as measured by $\mathrm{MIC}_{90}$ ) against individual CNS spp. derived from SSTIs (Table 4). Ozenoxacin was the most active agent against Staphylococcus capitis $(0.06 \mathrm{mg} / \mathrm{l})$ and $S$. lugdunensis $(0.015 \mathrm{mg} / \mathrm{l})$. Ozenoxacin was the fourth most active agent against $S$. epidermidis $(1 \mathrm{mg} / \mathrm{l})$ after retapamulin, daptomycin and tigecycline in order. Ozenoxacin and mupirocin were equal-second most active agents against $S$. haemolyticus $(0.25 \mathrm{mg} / \mathrm{l})$ after retapamulin. Ozenoxacin was the third most active agent against $S$. hominis $(1 \mathrm{mg} / \mathrm{l})$ after retapamulin and daptomycin.

Resistance rates to fusidic acid identified in several staphylococcal species ( $S$. aureus spp. [n $=2398]: 6.5 \%$; methicillin-resistant $S$. aureus [MRSA; $\mathrm{n}=1053$ ]: 8.5\%; methicillin-susceptible $S$. aureus [MSSA; $\mathrm{n}=1345$ ]: 5.1\%; and CNS spp. [n $=889$ ]: $24.6 \%$ ) were higher when antimicrobial activity was tested in isolates derived from SSTIs (S. aureus spp. [ $\mathrm{n}=1518]: 7.8 \%$; CNS spp. [ $\mathrm{n}=618]: 24.1 \%$; S. capitis $[\mathrm{n}=30]: 16.7 \%$; S. epidermidis $[\mathrm{n}=365]$ : 26.8\%; S. haemolyticus $[\mathrm{n}=76]: 26.3 \%$; and S. hominis $[\mathrm{n}=48]: 33.3 \%)$. Resistance rates to mupirocin in these same species were (S. aureus spp.: 4.8\%; MRSA: 9.0\%; MSSA: 1.6\%; and CNS spp.: 27.8\%); and in isolates derived from SSTIs were (S. aureus spp.: 4.7\%; CNS spp.: 27.7\%; S. capitis: 6.7\%; S. epidermidis: 40.5\%; S. haemolyticus: 5.3\%; and S. hominis: $22.9 \%)$. 
Table 3. Summary minimum inhibitory concentration data of ozenoxacin stratified by methicillin resistance/susceptibility and/or levofloxacin susceptibility/nonsusceptibility for Gram-positive bacteria (staphylococci, streptococci and enterococci).

\begin{tabular}{|c|c|c|c|c|c|}
\hline \multirow[t]{2}{*}{ Pathogen } & \multirow[t]{2}{*}{ Levofloxacin susceptibility } & \multirow[t]{2}{*}{$\mathbf{n}$} & \multicolumn{3}{|c|}{ Ozenoxacin MIC (mg/l) } \\
\hline & & & $\mathrm{MIC}_{50}$ & $\mathrm{MIC}_{90}$ & Range \\
\hline \multirow[t]{3}{*}{ Staphylococcus aureus } & All & 2398 & 0.004 & 0.25 & $\leq 0.001-2$ \\
\hline & Levofloxacin nonsusceptible & 877 & 0.12 & 0.25 & $0.002-2$ \\
\hline & Levofloxacin susceptible & 1521 & 0.004 & 0.004 & $\leq 0.001-2$ \\
\hline \multirow[t]{3}{*}{ - Methicillin-resistant S. aureus (MRSA) } & All & 1053 & 0.12 & 0.25 & $\leq 0.001-2$ \\
\hline & Levofloxacin nonsusceptible & 769 & 0.12 & 0.25 & $\leq 0.001-2$ \\
\hline & Levofloxacin susceptible & 284 & 0.004 & 0.008 & $0.002-2$ \\
\hline \multirow[t]{3}{*}{ - Methicillin-susceptible S. aureus (MSSA) } & All & 1345 & 0.004 & 0.008 & $\leq 0.001-2$ \\
\hline & Levofloxacin nonsusceptible & 108 & 0.12 & 1 & $0.004-2$ \\
\hline & Levofloxacin susceptible & 1237 & 0.004 & 0.004 & $\leq 0.001-0.5$ \\
\hline \multirow[t]{3}{*}{ Coagulase-negative staphylococci (CNS) } & All & 889 & 0.015 & 0.25 & $\leq 0.001-2$ \\
\hline & Levofloxacin nonsusceptible & 395 & 0.12 & 1 & $0.03-2$ \\
\hline & Levofloxacin susceptible & 494 & 0.008 & 0.015 & $\leq 0.001-0.12$ \\
\hline \multirow[t]{3}{*}{ - Methicillin-resistant CNS } & All & 500 & 0.12 & 1 & $\leq 0.001-2$ \\
\hline & Levofloxacin nonsusceptible & 346 & 0.12 & 1 & $0.03-2$ \\
\hline & Levofloxacin susceptible & 154 & 0.008 & 0.015 & $\leq 0.001-0.03$ \\
\hline \multirow[t]{3}{*}{ - Methicillin-susceptible CNS } & All & 389 & 0.008 & 0.12 & $0.002-2$ \\
\hline & Levofloxacin nonsusceptible & 49 & 0.12 & 2 & $0.03-2$ \\
\hline & Levofloxacin susceptible & 340 & 0.008 & 0.015 & $0.002-0.12$ \\
\hline \multirow[t]{3}{*}{ Staphylococcus capitis } & All & 53 & 0.008 & 0.12 & $0.002-0.12$ \\
\hline & Levofloxacin nonsusceptible & 13 & 0.06 & 0.12 & $0.03-0.12$ \\
\hline & Levofloxacin susceptible & 40 & 0.008 & 0.008 & $0.002-0.015$ \\
\hline \multirow[t]{3}{*}{ - Methicillin-resistant S. capitis } & All & 23 & 0.008 & 0.12 & $0.004-0.12$ \\
\hline & Levofloxacin nonsusceptible & 11 & 0.06 & 0.12 & $0.03-0.12$ \\
\hline & Levofloxacin susceptible & 12 & 0.008 & 0.008 & $0.004-0.008$ \\
\hline \multirow[t]{3}{*}{ - Methicillin-susceptible S. capitis } & All & 30 & 0.008 & 0.008 & $0.002-0.12$ \\
\hline & Levofloxacin nonsusceptible & 2 & 0.06 & 0.12 & $0.03-0.12$ \\
\hline & Levofloxacin susceptible & 28 & 0.008 & 0.008 & $0.002-0.15$ \\
\hline \multirow[t]{3}{*}{ Staphylococcus epidermidis } & All & 507 & 0.06 & 1 & $\leq 0.001-2$ \\
\hline & Levofloxacin nonsusceptible & 265 & 0.12 & 2 & $0.03-2$ \\
\hline & Levofloxacin susceptible & 242 & 0.008 & 0.015 & $\leq 0.001-0.03$ \\
\hline \multirow[t]{3}{*}{ - Methicillin-resistant S. epidermidis } & All & 337 & 0.12 & 1 & $\leq 0.001-2$ \\
\hline & Levofloxacin nonsusceptible & 237 & 0.12 & 2 & $0.03-2$ \\
\hline & Levofloxacin susceptible & 100 & 0.008 & 0.015 & $\leq 0.001-0.015$ \\
\hline \multirow[t]{3}{*}{ - Methicillin-susceptible S. epidermidis } & All & 170 & 0.008 & 0.12 & $0.002-2$ \\
\hline & Levofloxacin nonsusceptible & 28 & 0.12 & 2 & $0.06-2$ \\
\hline & Levofloxacin susceptible & 142 & 0.008 & 0.015 & $0.002-0.03$ \\
\hline \multirow[t]{3}{*}{ Staphylococcus haemolyticus } & All & 106 & 0.12 & 0.25 & $0.002-1$ \\
\hline & Levofloxacin nonsusceptible & 67 & 0.12 & 0.25 & $0.03-1$ \\
\hline & Levofloxacin susceptible & 39 & 0.004 & 0.008 & $0.002-0.008$ \\
\hline \multirow[t]{3}{*}{ - Methicillin-resistant S. haemolyticus } & All & 73 & 0.12 & 0.25 & $0.004-1$ \\
\hline & Levofloxacin nonsusceptible & 62 & 0.12 & 0.25 & $0.06-1$ \\
\hline & Levofloxacin susceptible & 11 & 0.004 & 0.008 & $0.004-0.008$ \\
\hline \multirow[t]{3}{*}{ - Methicillin-susceptible S. haemolyticus } & All & 33 & 0.008 & 0.12 & $0.002-0.25$ \\
\hline & Levofloxacin nonsusceptible & 5 & 0.12 & 0.25 & $0.03-0.25$ \\
\hline & Levofloxacin susceptible & 28 & 0.004 & 0.008 & $0.002-0.008$ \\
\hline
\end{tabular}


Table 3. Summary minimum inhibitory concentration data of ozenoxacin stratified by methicillin resistance/susceptibility and/or levofloxacin susceptibility/nonsusceptibility for Gram-positive bacteria (staphylococci, streptococci and enterococci) (cont.).

\begin{tabular}{|c|c|c|c|c|c|}
\hline \multirow[t]{2}{*}{ Pathogen } & \multirow[t]{2}{*}{ Levofloxacin susceptibility } & \multirow[t]{2}{*}{ n } & \multicolumn{3}{|c|}{ Ozenoxacin MIC (mg/l) } \\
\hline & & & $\mathrm{MIC}_{50}$ & $\mathrm{MIC}_{90}$ & Range \\
\hline \multirow[t]{3}{*}{ Staphylococcus hominis } & All & 94 & 0.008 & 0.25 & $0.002-2$ \\
\hline & Levofloxacin nonsusceptible & 33 & 0.12 & 2 & $0.03-2$ \\
\hline & Levofloxacin susceptible & 61 & 0.008 & 0.008 & $0.002-0.12$ \\
\hline \multirow[t]{3}{*}{ - Methicillin-resistant S. hominis } & All & 51 & 0.06 & 1 & $0.004-2$ \\
\hline & Levofloxacin nonsusceptible & 29 & 0.12 & 2 & $0.03-2$ \\
\hline & Levofloxacin susceptible & 22 & 0.008 & 0.015 & $0.004-0.015$ \\
\hline \multirow[t]{3}{*}{ - Methicillin-susceptible S. hominis } & All & 43 & 0.008 & 0.06 & $0.002-1$ \\
\hline & Levofloxacin nonsusceptible & 4 & 0.12 & 1 & $0.06-1$ \\
\hline & Levofloxacin susceptible & 39 & 0.008 & 0.008 & $0.002-0.12$ \\
\hline \multirow[t]{3}{*}{ Staphylococcus lugdunensis } & All & 63 & 0.015 & 0.015 & $0.004-0.25$ \\
\hline & Levofloxacin nonsusceptible & 3 & 0.25 & 0.25 & $0.12-0.25$ \\
\hline & Levofloxacin susceptible & 60 & 0.015 & 0.015 & $0.004-0.03$ \\
\hline \multirow[t]{3}{*}{ - Methicillin-resistant S. lugdunensis } & All & 2 & 0.008 & 0.12 & $0.008-0.12$ \\
\hline & Levofloxacin nonsusceptible & 1 & 0.12 & 0.12 & $0.12-0.12$ \\
\hline & Levofloxacin susceptible & 1 & 0.008 & 0.008 & $0.008-0.008$ \\
\hline \multirow[t]{3}{*}{ - Methicillin-susceptible S. Iugdunensis } & All & 61 & 0.015 & 0.015 & $0.004-0.25$ \\
\hline & Levofloxacin nonsusceptible & 2 & 0.25 & 0.25 & $0.25-0.25$ \\
\hline & Levofloxacin susceptible & 59 & 0.015 & 0.03 & $0.004-0.03$ \\
\hline \multirow[t]{3}{*}{ Staphylococcus pettenkoferi } & All & 14 & 0.5 & 0.5 & $0.004-1$ \\
\hline & Levofloxacin nonsusceptible & 10 & 0.5 & 1 & $0.12-1$ \\
\hline & Levofloxacin susceptible & 4 & 0.008 & 0.03 & $0.004-0.03$ \\
\hline \multirow[t]{3}{*}{ - Methicillin-resistant S. petteternkoferi } & All & 4 & 0.5 & 1 & $0.03-1$ \\
\hline & Levofloxacin nonsusceptible & 3 & 0.5 & 1 & $0.5-1$ \\
\hline & Levofloxacin susceptible & 1 & 0.03 & 0.03 & $0.03-0.03$ \\
\hline \multirow[t]{3}{*}{ - Methicillin-susceptible S. pettenkoferi } & All & 10 & 0.12 & 0.5 & $0.004-1$ \\
\hline & Levofloxacin nonsusceptible & 7 & 0.5 & 1 & $0.12-1$ \\
\hline & Levofloxacin susceptible & 3 & 0.008 & 0.008 & $0.004-0.008$ \\
\hline \multirow[t]{3}{*}{ - Staphylococcus warneri } & All & 16 & 0.008 & 0.015 & $0.004-0.015$ \\
\hline & Levofloxacin nonsusceptible & 3 & 0.008 & 0.015 & $0.008-0.015$ \\
\hline & Levofloxacin susceptible & 13 & 0.008 & 0.015 & $0.004-0.015$ \\
\hline$\beta$-haemolytic streptococci & All & 1210 & 0.03 & 0.03 & $0.004-2$ \\
\hline \multirow[t]{3}{*}{ - Streptococcus agalactiae } & All & 327 & 0.03 & 0.06 & $0.008-1$ \\
\hline & Levofloxacin nonsusceptible & 8 & 1 & 1 & $0.25-1$ \\
\hline & Levofloxacin susceptible & 319 & 0.03 & 0.06 & $0.008-0.12$ \\
\hline \multirow[t]{3}{*}{ - Streptococcus dysgalactiae } & All & 222 & 0.03 & 0.03 & $0.008-0.25$ \\
\hline & Levofloxacin nonsusceptible & 5 & 0.25 & 0.25 & $0.06-0.25$ \\
\hline & Levofloxacin susceptible & 217 & 0.015 & 0.03 & $0.008-0.25$ \\
\hline \multirow[t]{3}{*}{ - Streptococcus pyogenes } & All & 657 & 0.015 & 0.03 & $0.004-2$ \\
\hline & Levofloxacin nonsusceptible & 2 & 0.06 & 2 & $0.06-2$ \\
\hline & Levofloxacin susceptible & 655 & 0.015 & 0.03 & $0.004-0.12$ \\
\hline Viridans streptococci & All & 533 & 0.03 & 0.06 & $\leq 0.001-2$ \\
\hline - Streptococcus anginosus & All & 117 & 0.015 & 0.03 & $\leq 0.001-0.06$ \\
\hline - Streptococcus constellatus & All & 70 & 0.015 & 0.03 & $\leq 0.001-0.06$ \\
\hline - Streptococcus cristatus & All & 3 & 0.015 & 0.03 & $0.015-0.03$ \\
\hline - Streptococcus equinus & All & 6 & 0.015 & 0.06 & $0.015-0.06$ \\
\hline
\end{tabular}




\begin{tabular}{|c|c|c|c|c|c|}
\hline \multirow[t]{2}{*}{ Pathogen } & \multirow[t]{2}{*}{ Levofloxacin susceptibility } & \multirow[t]{2}{*}{$\mathrm{n}$} & \multicolumn{3}{|c|}{ Ozenoxacin MIC (mg/l) } \\
\hline & & & $\mathrm{MIC}_{50}$ & $\mathrm{MIC}_{90}$ & Range \\
\hline \multirow[t]{3}{*}{ - Streptococcus gallolyticus } & All & 60 & 0.03 & 0.06 & $0.008-2$ \\
\hline & Levofloxacin nonsusceptible & 4 & 0.12 & 2 & $0.06-2$ \\
\hline & Levofloxacin susceptible & 56 & 0.03 & 0.06 & $0.008-0.12$ \\
\hline - Streptococcus intermedius & All & 22 & 0.015 & 0.03 & $0.004-0.06$ \\
\hline \multirow[t]{3}{*}{ - Streptococcus mitis } & All & 65 & 0.03 & 0.06 & $0.015-0.5$ \\
\hline & Levofloxacin nonsusceptible & 2 & 0.25 & 0.5 & $0.25-0.5$ \\
\hline & Levofloxacin susceptible & 63 & 0.03 & 0.06 & $0.015-0.06$ \\
\hline \multirow[t]{3}{*}{ - Streptococcus oralis } & All & 60 & 0.03 & 0.06 & $0.015-0.25$ \\
\hline & Levofloxacin nonsusceptible & 2 & 0.25 & 0.25 & $0.25-0.25$ \\
\hline & Levofloxacin susceptible & 58 & 0.03 & 0.06 & $0.015-0.06$ \\
\hline \multirow[t]{3}{*}{ - Streptococcus parasanguinis } & All & 26 & 0.03 & 0.25 & $0.015-2$ \\
\hline & Levofloxacin nonsusceptible & 4 & 0.25 & 2 & $0.06-2$ \\
\hline & Levofloxacin susceptible & 22 & 0.03 & 0.03 & $0.015-0.06$ \\
\hline \multirow[t]{3}{*}{ - Streptococcus peroris } & All & 13 & 0.03 & 0.06 & $0.015-1$ \\
\hline & Levofloxacin nonsusceptible & 1 & 1 & 1 & $1-1$ \\
\hline & Levofloxacin susceptible & 12 & 0.03 & 0.06 & $0.015-0.06$ \\
\hline - Streptococcus salivarius & All & 41 & 0.03 & 0.06 & $0.015-0.06$ \\
\hline - Streptococcus sanguinis & All & 19 & 0.03 & 0.06 & $0.015-0.06$ \\
\hline \multirow[t]{5}{*}{ Streptococcus pneumoniae } & All & 927 & 0.03 & 0.03 & $\leq 0.001-2$ \\
\hline & Levofloxacin nonsusceptible & 11 & 0.25 & 2 & $0.06-2$ \\
\hline & Levofloxacin susceptible & 916 & 0.03 & 0.03 & $\leq 0.001-0.12$ \\
\hline & Penicillin nonsusceptible & 96 & 0.03 & 0.03 & $0.008-2$ \\
\hline & Penicillin susceptible & 831 & 0.03 & 0.03 & $\leq 0.001-0.5$ \\
\hline Enterococci & All & 166 & 0.06 & 1 & $\leq 0.001-2$ \\
\hline \multirow[t]{3}{*}{ - Enterococcus faecalis } & All & 159 & 0.06 & 1 & $\leq 0.001-2$ \\
\hline & Levofloxacin nonsusceptible & 48 & 1 & 1 & $0.06-2$ \\
\hline & Levofloxacin susceptible & 111 & 0.03 & 0.06 & $\leq 0.001-0.25$ \\
\hline
\end{tabular}

Streptococci \& enterococci

Ozenoxacin was highly active against levofloxacin nonsusceptible/susceptible streptococci and Enterococcus spp. (Table 3) with a $\mathrm{MIC}_{90}$ of $0.03 \mathrm{mg} / \mathrm{l}$ against combined $\beta$-haemolytic streptococci; 0.06 against combined viridans streptococci; $0.03 \mathrm{mg} / \mathrm{l}$ against $S$ pneumoniae; and $1 \mathrm{mg} / \mathrm{l}$ against enterococci including E. faecalis.

In SSTI-derived isolates (Table 4), ozenoxacin had the lowest or equal-lowest MIC 90 compared with 22 other antibacterial agents tested against the following bacteria: combined $\beta$-haemolytic streptococci, S. agalactiae, combined viridans streptococci, $S$. anginosus, $S$. constellatus, $S$. oralis and $S$. pneumoniae. The activity of ozenoxacin against remaining streptococci and enterococci was generally within one dilution of the most active compound. Against combined $\beta$-haemolytic streptococci, ozenoxacin had the lowest $\mathrm{MIC}_{90}(0.03 \mathrm{mg} / \mathrm{l})$ of all 23 antibacterial agents tested. Meropenem, penicillin, amoxicillin-clavulanic acid, ceftriaxone, cefuroxime and retapamulin each had a $\mathrm{MIC}_{90}$ of $0.06 \mathrm{mg} / \mathrm{l}$, while ampicillin, teicoplanin and tigecycline each had a MIC 90 of $0.012 \mathrm{mg} / 1$. MIC 90 values for all other agents were higher than that of ozenoxacin: $0.25 \mathrm{mg} / 1$ for moxifloxacin, daptomycin, piperacillintazobactam and trimethoprim-sulfamethoxazole; $0.5 \mathrm{mg} / 1$ for vancomycin; $1 \mathrm{mg} / 1$ for mupirocin, levofloxacin, ceftazidime and linezolid; $4 \mathrm{mg} / 1$ for clarithromycin; $16 \mathrm{mg} / 1$ for fusidic acid; and $64 \mathrm{mg} / 1$ for tetracycline and neomycin.

Against E. faecalis (Table 4), tigecycline ( $\mathrm{MIC}_{90}$ of $0.12 \mathrm{mg} / \mathrm{l}$ ) was the most active agent, followed by amoxicillinclavulanic acid and tetracycline (both $0.05 \mathrm{mg} / \mathrm{dl}$ ), then ozenoxacin and ampicillin (both $1 \mathrm{mg} / \mathrm{dl}$ ). 


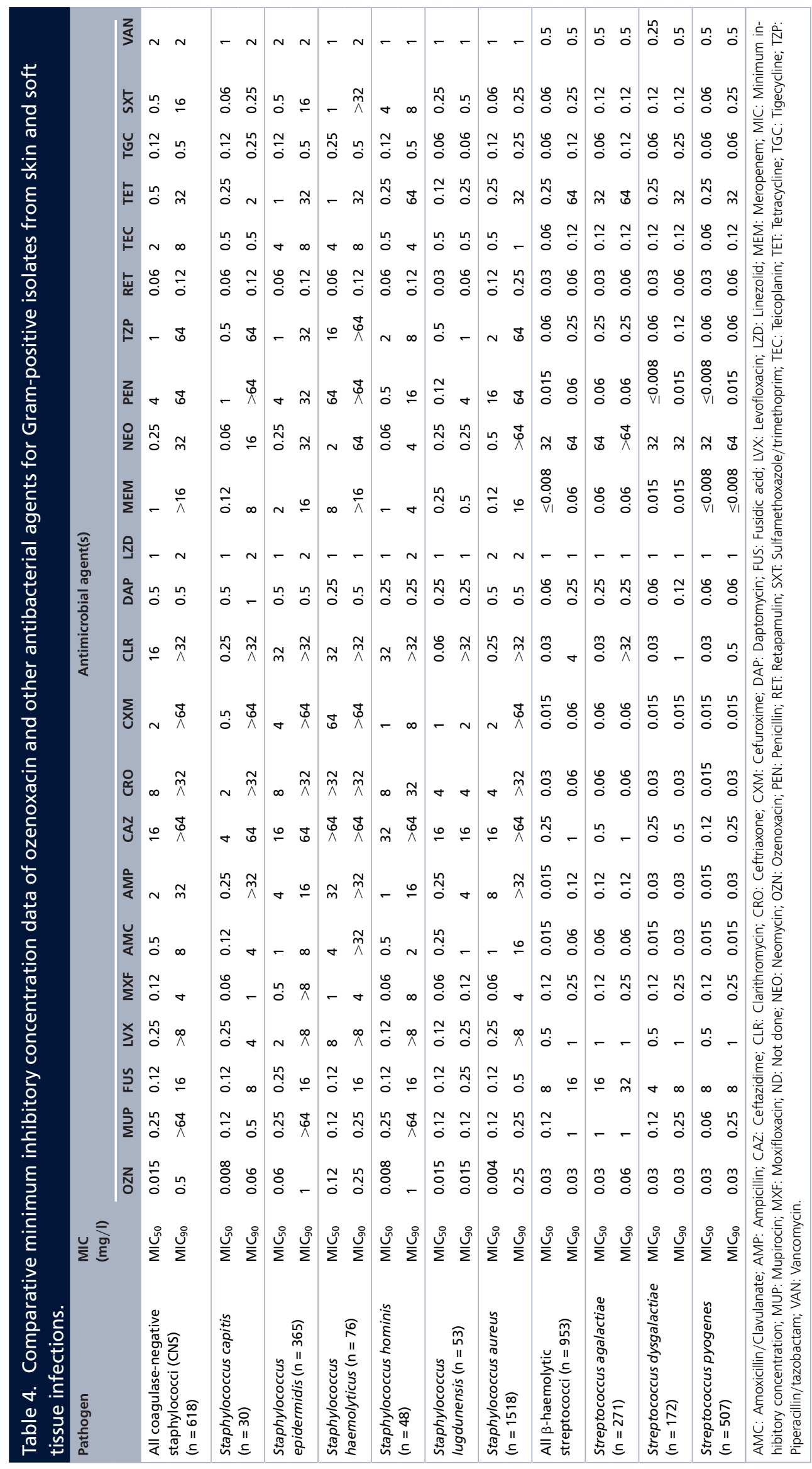




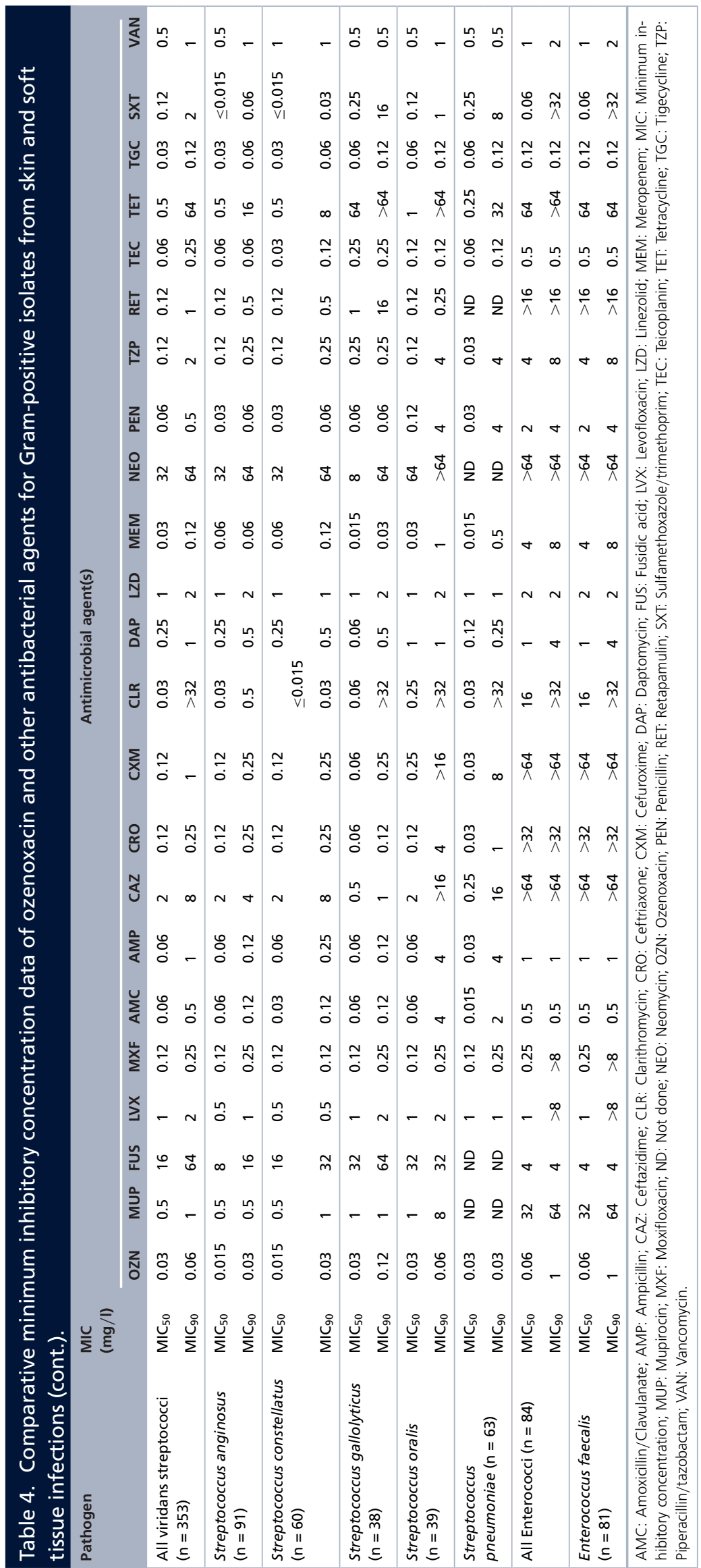


Table 5. Summary minimum inhibitory concentration data of ozenoxacin for Gram-negative bacteria.

\begin{tabular}{|c|c|c|c|c|}
\hline \multirow[t]{2}{*}{ Pathogen } & \multirow[t]{2}{*}{$n$} & \multicolumn{3}{|c|}{ Ozenoxacin MIC (mg/l) } \\
\hline & & $\mathrm{MIC}_{50}$ & $\mathrm{MIC}_{90}$ & Range \\
\hline All Enterobacteriaceae & 3190 & 0.25 & $>2$ & $0.004->2$ \\
\hline - Citrobacter braakii & 11 & 1 & $>2$ & $0.06->2$ \\
\hline - Citrobacter freundii & 133 & 0.25 & $>2$ & $0.03->2$ \\
\hline - Citrobacter koseri & 119 & 0.06 & 0.12 & $0.015->2$ \\
\hline - Klebsiella aerogenes & 158 & 0.25 & $>2$ & $0.03->2$ \\
\hline - Enterobacter asburiae & 21 & 0.12 & 0.5 & $0.03->2$ \\
\hline - Enterobacter cloacae & 219 & 0.12 & $>2$ & $0.015->2$ \\
\hline - Enterobacter kobei & 11 & 0.25 & $>2$ & $0.03->2$ \\
\hline - Escherichia coli & 1051 & 0.06 & $>2$ & $0.004->2$ \\
\hline - Klebsiella oxytoca & 197 & 0.25 & 1 & $0.03->2$ \\
\hline - Klebsiella pneumoniae & 544 & 0.25 & $>2$ & $0.008->2$ \\
\hline - Morganella morganii & 233 & 0.25 & $>2$ & $0.03->2$ \\
\hline - Salmonella spp. & 163 & 0.12 & 0.5 & $0.015->2$ \\
\hline - Serratia marcescens & 232 & 1 & $>2$ & $0.004->2$ \\
\hline - Shigella flexneri & 40 & 0.03 & 0.5 & $0.008->2$ \\
\hline -Shigella sonnei & 41 & 0.03 & 0.25 & $0.008->2$ \\
\hline \multicolumn{5}{|l|}{ Other Gram-negative bacteria } \\
\hline - Haemophilus spp. & 548 & 0.004 & 0.03 & $\leq 0.001-2$ \\
\hline - Haemophilus influenza & 470 & 0.004 & 0.015 & $\leq 0.001-1$ \\
\hline - Haemophilus parainfluenza & 73 & 0.03 & 0.25 & $\leq 0.001-2$ \\
\hline - Moraxella catarrhalis & 180 & 0.004 & 0.008 & $\leq 0.001-0.03$ \\
\hline - Neisseria gonorrheae & 13 & 0.12 & 1 & $\leq 0.001-2$ \\
\hline
\end{tabular}

\section{Gram-negative bacteria}

Enterobacteriaceae

Ozenoxacin had a $\mathrm{MIC}_{90}$ of more than $2 \mathrm{mg} / \mathrm{l}$ for combined species of the Enterobacteriaceae family, with values ranging from $0.12 \mathrm{mg} / \mathrm{l}$ for Citrobacter koseri, $0.25 \mathrm{mg} / \mathrm{l}$ for Shigella sonnei, $0.5 \mathrm{mg} / 1$ for Enterobacter asburiae and S. flexneri, $1 \mathrm{mg} / \mathrm{l}$ for Klebsiella oxytoca, and more than $2 \mathrm{mg} / \mathrm{l}$ for all remaining species (Table 5).

MIC values against Gram-negative bacteria derived from SSTIs were compared for ozenoxacin and 13 other antibacterial agents. Based on $\mathrm{MIC}_{90}$, ozenoxacin was the most active quinolone against combined Enterobacteriaceae, E. cloacae, E. coli, K. pneumoniae and Morganella morganii. In contrast, ozenoxacin was the least active quinolone against $C$. freundii, $C$. koseri, E. aerogenes, $K$. oxytoca and Serratia marcescens. However, the quinolones were generally of similar activity with $\mathrm{MIC}_{90}$ values within one or two dilutions of each other.

For combined species of Enterobacteriaceae, the most active compounds (as measured by $\mathrm{MIC}_{90}$ ) were meropenem $(0.06 \mathrm{mg} / \mathrm{l})$, tigecycline $(2 \mathrm{mg} / \mathrm{l})$ and ozenoxacin $(>2 \mathrm{mg} / \mathrm{l})$, followed by neomycin $(4 \mathrm{mg} / \mathrm{l})$, ciprofloxacin $(>4 \mathrm{mg} / \mathrm{l})$, levofloxacin and moxifloxacin (each $>8 \mathrm{mg} / \mathrm{l}$ ). All other antibacterial agents had $\mathrm{MIC}_{90}$ values of 32 to $>128 \mathrm{mg} / \mathrm{l}$ (Table 6$)$.

Other Gram-negative bacteria

For other Gram-negative bacteria (Table 5), ozenoxacin was highly active against Haemophilus spp. (MIC 90 of $0.03 \mathrm{mg} / \mathrm{l}), H$. influenzae $(0.015 \mathrm{mg} / \mathrm{l})$ and Moraxella catarrhalis $(0.008 \mathrm{mg} / \mathrm{l})$, and was somewhat less active against $H$. parainfluenzae $(0.25 \mathrm{mg} / \mathrm{l})$ and Neisseria gonorrhoeae $(1 \mathrm{mg} / \mathrm{l})$, although it was tested on only $13 N$. gonorrhoeae isolates.

Ozenoxacin and levofloxacin $\left(\mathrm{MIC}_{90}\right.$ of $\left.0.015 \mathrm{mg} / \mathrm{l}\right)$ were the second most active antibacterial agents against $H$. influenzae after ceftriaxone $(0.008 \mathrm{mg} / \mathrm{l})$, followed by piperacillin/tazobactam $(\leq 0.03 \mathrm{mg} / \mathrm{l})$, moxifloxacin $(0.03 \mathrm{mg} / \mathrm{l})$, meropenem $(0.06 \mathrm{mg} / \mathrm{l})$ and ceftazidime $(0.12 \mathrm{mg} / \mathrm{l})$; all other antibacterial agents had MIC 90 values of $0.5-16 \mathrm{mg} / \mathrm{l}$. 


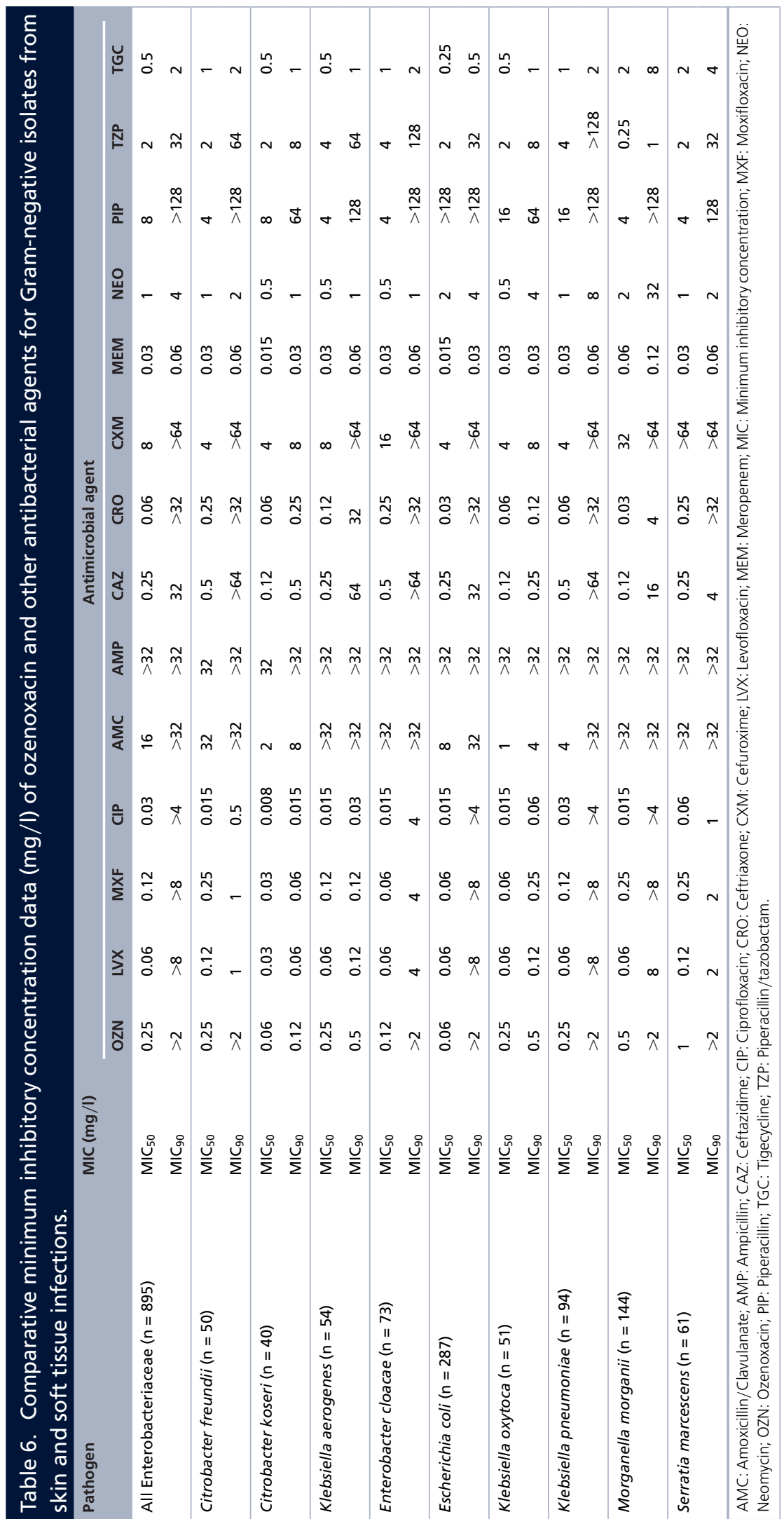


All quinolones were less active against $H$. parainfluenzae than $H$. influenzae. Ceftriaxone $\left(\mathrm{MIC}_{90}\right.$ of $\left.0.008 \mathrm{mg} / \mathrm{l}\right)$ was the most active antibacterial agent against this species, followed by ceftazidime and meropenem $(0.06 \mathrm{mg} / \mathrm{l})$, levofloxacin $(0.12 \mathrm{mg} / \mathrm{l})$, then ozenoxacin, moxifloxacin and piperacillin/tazobactam $(0.25 \mathrm{mg} / \mathrm{l})$; all other antibacterial agents had $\mathrm{MIC}_{90}$ values of $1-16 \mathrm{mg} / \mathrm{l}$.

Ozenoxacin and meropenem were the most active agents against $M$. catarrhalis $\left(\mathrm{MIC}_{90}\right.$ of $\leq 0.008 \mathrm{mg} / \mathrm{l}$ ), followed by piperacillin/tazobactam $(\leq 0.03 \mathrm{mg} / \mathrm{l})$, levofloxacin and moxifloxacin $(0.06 \mathrm{mg} / \mathrm{l})$, and clarithromycin $(0.12 \mathrm{mg} / \mathrm{l})$; all other agents had $\mathrm{MIC}_{90}$ values of $0.25-16 \mathrm{mg} / \mathrm{l}$.

\section{Discussion}

A previously published study that compared the antimicrobial activity of ozenoxacin versus 17 antibacterial agents in more than 2000 Gram-positive clinical isolates from SSTIs showed that ozenoxacin is a potent antimicrobial agent against both staphylococci and streptococci, irrespective of levofloxacin susceptibility status [14]. The current comparative study examined the antibacterial activity of ozenoxacin in Gram-positive and Gram-negative clinical isolates derived mainly from SSTIs, RTIs and UTIs, which were stratified according to their methicillin and levofloxacin susceptibility status. The antibacterial activity of ozenoxacin was compared with that of 22 other antibacterial agents against approximately 4500 Gram-positive SSTI isolates, and with that of 13 other antibacterial agents against approximately 900 Gram-negative SSTIs isolates and Gram-negative isolates from other sources (e.g., RTIs, UTIs, etc).

Against Gram-positive isolates from SSTIs, ozenoxacin was highly active against staphylococci and streptococci, confirming the results of previously published comparative studies [14]. Ozenoxacin had a lower MIC g0 $_{90}$ than mupirocin and fusidic acid against the CNS isolates S. capitis, S. epidermidis, S. hominis and S. lugdunensis, and against combined $\beta$-haemolytic streptococci, combined viridans streptococci, all other subspecies of streptococci, and enterococci. Ozenoxacin was equally active as mupirocin (as measured by $\mathrm{MIC}_{90}$ ) against $S$. haemolyticus and $S$. aureus. These results confirm and extend the previously reported results showing higher activity of ozenoxacin compared with mupirocin and fusidic acid against staphylococci and streptococci [14].

Clinical breakpoints of antibiotics are calculated based on MIC distributions, and pharmacokinetic and pharmacodynamic data (area under the curve of plasma concentrations). Because topical antibiotics are minimally absorbed, clinical breakpoints cannot be determined. Instead, combined MIC distributions from multiple sources and time periods are used to define epidemiological cutoffs that provide an indication of MICs for organisms with acquired or mutational resistance mechanisms. The inferred breakpoint for mupirocin is $1 / 256 \mathrm{mg} / \mathrm{l}$ and applies to topical nasal administration three-times daily. It is based on microbiological data (epidemiological cutoffs) and clinical experience [20]. In the case of fusidic acid, breakpoints for Staphylococcus spp. are $S \leq 1 \mathrm{mg} / \mathrm{l}$ and R $>1 \mathrm{mg} / \mathrm{l}$ for an oral or IV dosage of $500 \mathrm{mg}$ two- to three-times daily. The breakpoints are based on pharmacokinetic data, microbiological data and clinical experience [21]. In the current study, BSAC disk diffusion breakpoints that are harmonized with the European Committee on Antimicrobial Testing were used for susceptibility testing of mupirocin and fusidic acid [18]. Disk diffusion tests with ozenoxacin have yet to be conducted but are planned. According to the BSAC breakpoints in this study, the resistance rate of $S$. aureus to mupirocin and fusidic acid was higher in MRSA isolates than in MSSA isolates (9.0 and 1.6\% for mupirocin and 8.5 and $5.1 \%$ for fusidic acid, respectively).

Fusidic acid, mupirocin or retapamulin are the main topical treatments recommended in Europe for treatment of impetigo [22], although resistance of staphylococcal strains to these agents is becoming a concern. Resistance to fusidic acid [23-27] and mupirocin [28-30] has been reported in Europe and may be increasing. Investigators in Greece reported a sevenfold increase from 2013 to 2016 in the rate of mupirocin-resistant $S$. aureus strains among community-associated staphylococcal infections (mainly impetigo cases), concurrent with a 1.9-fold increase in resistance rates to fusidic acid [31], highlighting the need for alternatives. Ozenoxacin's dual inhibitory activity against DNA gyrase and topoisomerase IV, in addition to other characteristics such as a mutant prevention concentration below that in skin, appear to protect it from the development of resistance [10].

Elsewhere, a Japanese comparative study of ozenoxacin with other antibacterial agents against cutaneous bacterial isolates from pediatric patients reported $\mathrm{MIC}_{90}$ values for ozenoxacin of $0.12, \leq 0.06$ and $\leq 0.06 \mathrm{mg} / \mathrm{l}$ against MRSA, MSSA and S. pyogenes isolates, respectively [32]. Respective $\mathrm{MIC}_{90}$ values for gentamicin against MRSA, MSSA and S. pyogenes isolates in this same Japanese study were more than 128, 64 and $4 \mathrm{mg} / \mathrm{l}$ for SSTIs from adults and 128,16 and $4 \mathrm{mg} / \mathrm{l}$ for SSTIs from children. Gentamicin is used topically to treat SSTIs in some countries 
but was not included in the present study. The Japanese results highlight that ozenoxacin is markedly more active than gentamicin against the most common Gram-positive bacteria found in SSTIs.

Another Japanese study showed that MICs of ozenoxacin against three levofloxacin-susceptible Propionibacterium acnes strains (MIC of levofloxacin; $\leq 4 \mu \mathrm{g} / \mathrm{ml}$ ) and three levofloxacin-resistant $P$. acnes strains (MIC of levofloxacin; $\geq 8 \mu \mathrm{g} / \mathrm{ml}$ ) ranged from 0.03 to $0.06 \mu \mathrm{g} / \mathrm{ml}$ and from 0.25 to $0.5 \mu \mathrm{g} / \mathrm{ml}$, respectively [33]. These results suggest that ozenoxacin has a potent bactericidal activity against both levofloxacin-susceptible and levofloxacin-resistant $P$. acnes, a microorganism not included in the present study. A $2 \%$ lotion formulation of ozenoxacin has been approved since 2015 in Japan for treatment of inflamed acne.

In this study, the quinolones (ozenoxacin, ciprofloxacin, levofloxacin and moxifloxacin) exhibited comparable activity against Enterobacteriaceae, whereas ozenoxacin was the most active quinolone against E. cloacae, E. coli, K. pneumoniae and M. morganii. Ozenoxacin had lower activity than other quinolones against C. freundii, C. koseri, $K$. aerogenes, $K$. oxytoca and $S$. marcescens, although the differences were relatively small.

As SSTIs are caused mainly by Gram-positive cocci (especially S. aureus and S. pyogenes), the numbers of Gramnegative isolates derived exclusively from SSTIs were insufficient to compare ozenoxacin with other antibacterial agents. Moreover, other commonly used topical antibiotics such as mupirocin [34] and fusidic acid [35] have no or limited activity against Gram-negative isolates. The vast majority of Haemophilus spp. and M. catarrhalis isolates were derived from RTIs. Nevertheless, irrespective of the source, ozenoxacin was highly active against all Haemophilus spp. $(0.03 \mathrm{mg} / \mathrm{l}), H$. influenzae $(0.015 \mathrm{mg} / \mathrm{l})$ and $M$. catarrhalis (MIC 90 of $0.008 \mathrm{mg} / \mathrm{l})$, and was somewhat less active against $H$. parainfluenzae $(0.25 \mathrm{mg} / \mathrm{l})$.

\section{Conclusion}

In conclusion, ozenoxacin was shown to be a potent antibacterial agent against Gram-positive (staphylococci and streptococci) isolates and showed activity against some Gram-negative isolates. The high activity of ozenoxacin in staphylococcal and streptococcal spp. including methicillin and quinolone-resistant strains most commonly associated with childhood impetigo suggests that ozenoxacin may be a valuable alternative to other topical antibiotics in the eradication of these same bacterial species derived from SSTIs.

\section{Summary points}

- Ozenoxacin, a novel nonfluorinated quinolone with bactericidal activity, is approved in the USA, Canada and 12 EU countries as a $1 \%$ cream formulation for treatment of impetigo and in Japan as a $2 \%$ lotion formulation for treatment of superficial skin infections and acne.

- During development of ozenoxacin, surveillance studies compared its in vitro antibacterial activity with that of other topical and systemic antibacterial agents.

- In 2010, a total of 10,054 Gram-positive and Gram-negative isolates were collected from 128 centers worldwide; isolates were derived mainly from skin and soft tissue infections (SSTIs; $44.8 \%$ ) and respiratory tract infections (38.1\%).

- Minimum inhibitory concentrations were determined for 23 and 13 antimicrobial agents, respectively, using standard broth microdilution methods.

- Ozenoxacin exhibited high in vitro activity against susceptible, and methicillin- or levofloxacin-resistant, Gram-positive bacteria (Staphylococcus aureus, coagulase-negative staphylococci, $\beta$-haemolytic streptococci, viridans streptococci, enterococci and Streptococcus pneumoniae isolates).

- In the case of Gram-positive SSTI isolates, ozenoxacin was generally more active than mupirocin or fusidic acid and other quinolones.

- Ozenoxacin showed one or two dilutions less activity against Enterobacteriaceae isolates, except for Escherichia coli, than other quinolones tested.

- In the case of Gram-negative SSTI isolates, no comparisons of ozenoxacin were made with mupirocin and fusidic acid as these antibiotics are not active against these bacteria.

- Ozenoxacin is a potent antimicrobial agent mainly against susceptible and resistant strains of Gram-positive isolates (staphylococci and streptococci), and shows activity against some Gram-negative isolates.

Financial \& competing interests disclosure

The studies were supported by Ferrer Internacional, Barcelona, Spain. I Morrissey is an employee of IHMA Europe who has received payment from numerous pharmaceutical companies to perform in vitro research on antimicrobial activity. R Canton, J Vila, M 
Garcia-Castillo and Y López have received research grants from Ferrer Internacional. D Gargallo-Viola is a former employee of Ferrer Internacional. I Zsolt is a current employee of Ferrer Internacional. The authors have no other relevant affiliations or financial involvements with any organization or entity with a financial interest in or financial conflict with the subject matter or materials discussed in the manuscript apart from those disclosed.

Writing assistance was provided by Content Ed Net (Madrid, Spain) with funding from Ferrer International SA (Barcelona, Spain).

\section{Ethical conduct of research}

The authors state that they have obtained appropriate institutional review board approval or have followed the principles outlined in the Declaration of Helsinki for all human or animal experimental investigations.

\section{Open access}

This work is licensed under the Attribution-NonCommercial-NoDerivatives 4.0 Unported License. To view a copy of this license, visit http://creativecommons.org/licenses/by-nc-nd/4.0/

\section{References}

Papers of special note have been highlighted as: $\bullet$ of interest; $\bullet \bullet$ of considerable interest

1. Bolognia J, Jorizzo JL, Schaffer JV. Bacterial diseases. In: Dermatology (3rd Edition). Bolognia J, Jorizzo JL, Schaffer JV (Eds). Saunders, PA, USA, 1187-1189 (2012).

2. Sladden MJ, Johnston GA. Common skin infections in children. BMJ 329, 95-99 (2004).

3. Abeck D. Staphylococcal and streptococcal diseases. In: Braun-Falcos Dermatology. Burgdorf W, Plewig G, Wolff HH, Landthaler M (Eds). Springer-Verlag, Berlin, Heidelberg, Germany, 114-139 (2009).

4. Garbe C, Wolf G. Topical therapy. In: Braun-Falco's Dermatology. Burgdorf W, Plewig G, Wolff HH, Landthaler M (Eds). Springer-Verlag, Berlin, Heidelberg, Germany, 1549-1572 (2009).

5. Edge R, Argáez C (Eds). Topical antibiotics for impetigo: a review of the clinical effectiveness and guidelines [Internet]. Ottawa (ON): Canadian Agency for Drugs and Technologies in Health. CADTH Rapid Response Reports (2017). www.cadth.ca/sites/default/files/pdf/htis/2017/RC0851\%20Topical\%20Antibiotics\%20for\%20Impetigo\%20Final.pdf

6. Yamakawa T, Mitsuyama J, Hayashi K. In vitro and in vivo antibacterial activity of T-3912, a novel non-fluorinated topical quinolone. J. Antimicrob. Chemother. 49(3), 455-465 (2002).

7. Cipher Pharmaceuticals Receives Health Canada Approval of OZANEX ${ }^{T M}$ (ozenoxacin cream 1\%) (2019). www.newswire.ca/news-relea ses/cipher-pharmaceuticals-receives-health-canada-approval-of-ozanex-ozenoxacin-cream-1-621597383.html

8. MHRA. Summary of product characteristics: ozadub $10 \mathrm{mg} / \mathrm{g}$ cream. Ozenoxacin (2019). www.mhra.gov.uk/spc-pil/?subsName=OZENOXACIN\&pageID=SecondLevel

9. Health Products Regulatory Authority. Summary of product characteristics: dubine $10 \mathrm{mg} / \mathrm{g}$ cream. Ozenoxacin. (2019). www.hpra.ie/ homepage/medicines/medicines-information/find-a-medicine/results?query=OZENOXACIN\&field=ACTIVESUBSTANCES

10. Vila J, Hebert AA, Torrelo A et al. Ozenoxacin: a review of preclinical and clinical efficacy. Expert Rev. Anti. Infect. Ther. 17(3), 159-168 (2019).

-• Comprehensive review of the microbiology, pharmacodynamic and pharmacokinetic properties of ozenoxacin, and its clinical and microbiological efficacy in impetigo.

11. Gropper S, Albareda N, Chelius K et al. Ozenoxacin 1\% cream in the treatment of impetigo: a multicenter, randomized, placebo- and retapamulin-controlled clinical trial. Future Microbiol. 9, 1013-1023 (2014).

-• Phase III clinical trial demonstrating the efficacy and safety of ozenoxacin $1 \%$ cream for treatment of impetigo.

12. Rosen $\mathrm{T}$, Albareda $\mathrm{N}$, Rosenberg $\mathrm{N}$ et al. Efficacy and safety of ozenoxacin cream for treatment of adult and pediatric patients with impetigo: a randomized clinical trial. JAMA Dermatol. 154, 806-813 (2018).

-• Phase III clinical trial demonstrating the efficacy and safety of ozenoxacin $1 \%$ cream for treatment of impetigo.

13. López $\mathrm{Y}$, Tato M, Espinal $\mathrm{P}$ et al. In vitro activity of ozenoxacin against quinolone-susceptible and quinolone-resistant gram-positive bacteria. Antimicrob. Agents Chemother. 57, 6389-6392 (2013).

14. Cantón R, Morrissey I, Vila J et al. Comparative in vitro antibacterial activity of ozenoxacin against Gram-positive clinical isolates. Future Microbiol. 13, 3-19 (2018).

- Comparison of the in vitro activity of ozenoxacin versus other antibacterial agents against Gram-positive clinical isolates from skin and soft tissue infections.

15. Clinical and Laboratory Standards Institute (CLSI). Methods for Antimicrobial Dilution and Disk Susceptibility Testing of Infrequently Isolated or Fastidious Bacteria; Approved Guideline. CLSI Document M45-A. CLSI, PA, USA (2006).

16. Clinical and Laboratory Standards Institute (CLSI). Methods for Dilution Antimicrobial Susceptibility Tests for Bacteria That Grow Aerobically; Approved Standard - Eighth Edition. CLSI Document M7-A8. CLSI, PA, USA (2009). 
17. Clinical and Laboratory Standards Institute (CLSI). Performance Standards for Antimicrobial Susceptibility Testing; Twentieth Informational Supplement. CLSI Document M100-S20. CLSI, PA, USA (2010).

18. British Society for Antimicrobial Chemotherapy. BSAC Methods for Antimicrobial Susceptibility Testing. Version 9.1 March 2010 (2010). http://bsac.org.uk/wp-content/uploads/2012/02/Version_9.1_March_2010_final-v2.pdf

19. Clinical and Laboratory Standards Institute (CLSI). Performance Standards for Antimicrobial Susceptibility Testing: Twenty-First Informational Supplement. CLSI Document M100-S21, PA, USA (2011).

20. European Committee on Antimicrobial Susceptibility Testing. Mupirocin: rationale for the clinical breakpoints, version 1.0, 2010. www.eucast.org

21. European Committee on Antimicrobial Susceptibility Testing. Fusidic acid: rationale for the clinical breakpoints, version 1.0, 2010. www.eucast.org

22. van Bijnen EM, Paget WJ, den Heijer CD et al. Primary care treatment guidelines for skin infections in Europe: congruence with antimicrobial resistance found in commensal Staphylococcus aureus in the community. BMC Fam. Pract. 15, 175 (2014).

23. Howden BP, Grayson ML. Dumb and dumber - the potential waste of a useful antistaphylococcal agent: emerging fusidic acid resistance in Staphylococcus aureus. Clin. Infect. Dis. 42, 394-400 (2006).

24. O'Neill AJ, Larsen AR, Skov R et al. Characterization of the epidemic European fusidic acid-resistant impetigo clone of Staphylococcus aureus. J. Clin. Microbiol. 45, 1505-1510 (2007).

25. Denton M, O'Connell B, Bernard P et al. The EPISA study: antimicrobial susceptibility of Staphylococcus aureus causing primary or secondary skin and soft tissue infections in the community in France, the UK and Ireland. J. Antimicrob. Chemother. 61, 586-588 (2008).

26. Alsterholm M, Flytström I, Bergbrant IM et al. Fusidic acid-resistant Staphylococcus aureus in impetigo contagiosa and secondarily infected atopic dermatitis. Acta Derm. Venereol. 90, 52-57 (2010).

27. Castanheira M, Watters AA, Mendes RE et al. Occurrence and molecular characterization of fusidic acid resistance mechanisms among Staphylococcus spp. from European countries (2008). J. Antimicrob. Chemother. 65, 1353-1358 (2010).

28. Rossney A, O’Connell S. Emerging high-level mupirocin resistance among MRSA isolates in Ireland. Euro. Surveill. 13, 8084 (2008).

29. Sareyyupoglu B, Ozyurt M, Haznedaroglu T et al. Detection of methicillin and mupirocin resistance in staphylococcal hospital isolates with a touchdown multiplex polymerase chain reaction. Folia Microbiol. (Praha) 53, 363-367 (2008).

30. Desroches M, Potier J, Laurent F et al. Prevalence of mupirocin resistance among invasive coagulase-negative staphylococci and methicillin-resistant Staphylococcus aureus (MRSA) in France: emergence of a mupirocin-resistant MRSA clone harbouring mupA. J. Antimicrob. Chemother. 68, 1714-1717 (2013).

31. Doudoulakakis A, Spiliopoulou I, Spyridis N et al. Emergence of a Staphylococcus aureus clone resistant to mupirocin and fusidic acid carrying exotoxin genes and causing mainly skin infections. J. Clin. Microbiol. 55, 2529-2537 (2017).

- Greek investigators describe emergence of a Staphylococcus aureus clone resistant to mupirocin and fusidic acid.

32. Kanayama S, Ikeda F, Okamoto K et al. In vitro antimicrobial activity of ozenoxacin against methicillin-susceptible Staphylococcus aureus, methicillin-resistant $S$. aureus and Streptococcus pyogenes isolated from clinical cutaneous specimens in Japan. J. Infect. Chemother. 22, 720-723 (2016).

- In vitro antibacterial activity of ozenoxacin against cutaneous isolates in Japan.

33. Kanayama S, Okamoto K, Ikeda F et al. Bactericidal activity and post-antibiotic effect of ozenoxacin against Propionibacterium acnes. J. Infect. Chemother. 23(6), 374-380 (2017).

- In vitro antibacterial activity of ozenoxacin against Propionibacterium acnes.

34. Ward A, Campoli-Richards DM. Mupirocin. A review of its antibacterial activity, pharmacokinetic properties and therapeutic use. Drugs 32(5), 425-444 (1986).

35. Verbist L. The antimicrobial activity of fusidic acid. J. Antimicrob. Chemother. 25(Suppl. B), 1-5 (1990). 
